\title{
Proteasomal degradation unleashes the pro-death activity of androgen receptor
}

\author{
Bradley Godfrey ${ }^{1}$, Yuting Lin ${ }^{1}$, Jeffery Larson ${ }^{2}$, Bonnie Haferkamp ${ }^{1}$, Jialing Xiang ${ }^{1}$ \\ ${ }^{I}$ Department of Biological, Chemical, and Physical Sciences, Illinois Institute of Technology, 3101 South Dearborn Street, Chi- \\ cago, IL 60616, USA; ${ }^{2}$ Department of Biomedical Engineering, Illinois Institute of Technology, 3101 South Dearborn Street, Chi- \\ cago, IL 60616, USA
}

Androgen receptor (AR) is able to promote stress-induced cell death independently of its transcription activity in androgen-independent prostate cancer cells. Yet, the underlying mechanism is incompletely understood. Here, we report that stress-induced proteasomal degradation of $A R$ contributes to its pro-death activity. Upon exposure to ultraviolet light and staurosporine, AR underwent proteasomal degradation. Blockade of AR degradation significantly suppressed stress-induced apoptosis in androgen-independent prostate cancer cells. Ectopic expression of the AR N-terminal (AR-N) domain, which lacks DNA- and ligand-binding abilities, led to cell death without any additional death stimuli. Truncation analysis revealed that AR-N domain contains several sub-domains that regulate the prodeath activity of $A R$, specifically the first 105 amino acids, which function as a minimal pro-death domain acting upstream of caspases. The pro-apoptotic activity of AR $\mathrm{N}$-terminal fragments was suppressed by ectopic expression of Bcl-2 or selected caspase inhibitors. Thus, our results reveal a novel mechanism by which AR promotes stressinduced cell death in androgen-independent prostate cancer cells.

Keywords: Androgen receptor; apoptosis; proteasomal degradation; pro-death domain

Cell Research (2010) 20:1138-1147. doi:10.1038/cr.2010.65; published online 18 May 2010

\section{Introduction}

Androgen receptor (AR) is a member of the steroid hormone receptor superfamily [1-3]. Upon binding to androgen, AR translocates into the nucleus, where it activates androgen target genes involved in cell proliferation and cell survival, or in the development of prostate cancer $[1,4,5]$. In the absence of androgen, AR resides in the cytoplasm $[6,7]$. Like other steroid hormone receptors, AR consists of three major functional domains: the $\mathrm{N}$-terminal transactivation domain, the $\mathrm{C}$-terminal DNA-binding domain (DBD), and the ligand-binding domain (LBD) $[3,8]$. The N-terminal transactivation domain contains several regulatory elements, such as AF1 (activation function 1) [9, 10], and post-translational modification sites for phosphorylation [11-13] and ubiq-

Correspondence: Jialing Xiang

Tel: +1-312-567-3491; Fax: +1-312-567-3494

E-mail: Xiang@iit.edu

Received 1 April 2010; revised 18 April 2010; accepted 19 April 2010; published online 18 May 2010 uitination $[14,15]$. This suggests that the AR N-terminal $(A R-N)$ region may be involved in regulation of $A R$ functions in addition to its transcriptional activity [16].

Emerging evidence suggests that androgen and AR play a critical role in regulation of cell death [17-21]. We have shown that AR augments stress-induced cell death independently of its transcription activity, at least in part, through promotion of Bax mitochondrial translocation [22]. It has been postulated that AR cytotoxicity may be correlated with its N-terminal expanded polyglutamine (polyQ) repeats $[23,24]$. In addition, degradation of AR by several proteases, including serine protease, caspase, and calpain, has been reported to associate with AR transactivation activity and cell viability [25-27]. For example, inhibition of calpain-mediated AR degradation by calpain inhibitors induces cell death, although the mechanism is unknown [25-27]. Here, we report that proteasomal degradation of AR is involved in cell death induced by ultraviolet (UV) light and staurosporine (STS) in androgen-independent prostate cancer cells. The AR-N domain, which lacks both DNA- and ligand-binding abilities, is capable of inducing cell death in the absence 
of any additional death stimuli and its pro-death activity subjects to positive or negative regulation by different sub-domains within the AR-N domain.

\section{Results}

Proteasomal degradation of AR promotes stress-induced cell death in androgen-independent prostate cancer $L N$ CaP 104-R1 cells

Previously, we have shown that AR is required for UV-induced cell death independently of its transcription activity in androgen-independent prostate cancer cells [22]. Silencing of AR by siRNA in AR-positive prostate cancer cells renders cells resistant to UV-induced cell death, while restoration of AR in AR-negative prostate cancer cells sensitizes cells to UV-induced cell death [22]. However, the underlying mechanism is incompletely understood. To determine whether post-translational modification of AR is involved in its pro-death action, androgen-independent and AR-positive LNCaP 104-R1 cells were irradiated with UV. As expected, cell death occurred $\sim 12-16 \mathrm{~h}$ post-UV irradiation (Figure 1A), consistent with the previous report [22]. Immunoblotting analysis with anti-AR antibody revealed that UV irradiation significantly reduced expression levels of endogenous AR proteins in a time-dependent manner (Figure 1B). Interestingly, upon UV irradiation, AR protein levels were reduced as early as $2 \mathrm{~h}$ after irradiation, and were only about $60 \%$ left at $8 \mathrm{~h}$ (Figure 1B). This suggests that AR degradation occurred prior to the onset of UV-induced cell death, which was detectable $12 \mathrm{~h}$ after UV treatment (Figure 1A).

To determine how UV induces the reduction of AR proteins and its relationship with UV-induced cell death, prostate cancer 104-R1 cells were pre-treated with a panel of protease inhibitors, including a proteasome inhibitor (MG-132), caspases inhibitors (YVAD for caspase-1, DEVD-fmk for caspase-3, LEHD-fmk for caspase-9,
A

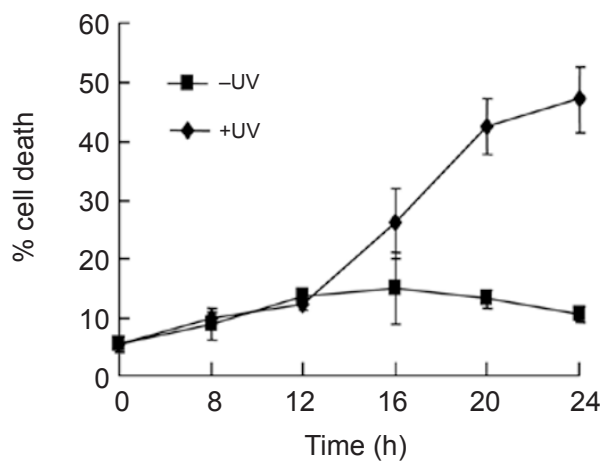

B

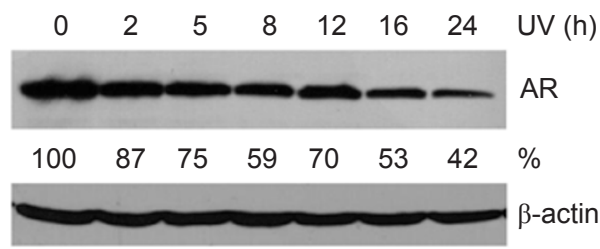

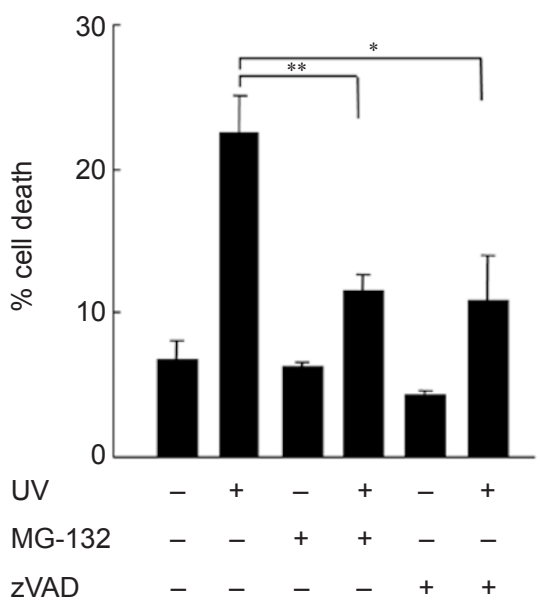

C

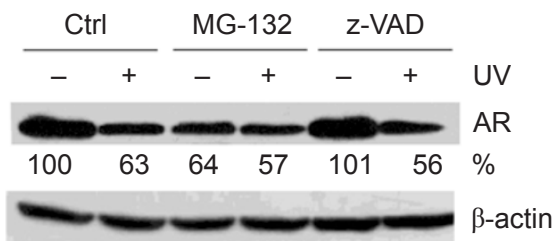

Figure 1 Proteasome-mediated degradation of AR promotes UV-irradiated apoptosis. (A) Androgen-independent LNCaP 104R1 cells were treated with or without UV irradiation, and cell death was monitored at the time periods as indicated. (B) Cell lysates were collected from a parallel experiment in A, and AR protein levels were analyzed by immunoblotting using anti-AR antibody. The percentage of AR reduction was normalized by $\beta$-actin. (C and D) 104-R1 cells were pre-treated with or without MG-132 (10 $\mu \mathrm{M})$ or z-VAD-fmk $(50 \mu \mathrm{M})$ for $2 \mathrm{~h}$, followed by UV irradiation, and incubated for another $16 \mathrm{~h}$. AR protein levels were analyzed by immunoblotting using anti-AR antibody. The percentage of AR reduction was quantified, as described in $\mathbf{B}$ (C). Cell death was monitored by trypan blue exclusion (D). Error bars indicate SD. ${ }^{*} P<0.05$; $* * P<0.01$ by $t$-test. Data shown are representative of three independent experiments. 
and z-VAD-fmk for pan-caspase), calpain inhibitors (calpain I and II, and calpastatin), serine protease inhibitors (leupeptin and aprotinin), and a cysteine protease inhibitor (E-64). Among the inhibitors tested, only the proteasome inhibitor MG-132 significantly blocked UVinduced reduction of AR proteins (Figure 1C). Under the same conditions, UV-induced cell death was inhibited by MG-132, as well as the pan-caspase inhibitor z-VADfmk (Figure 1D) and caspase-3 inhibitor DEVD-fmk (data not shown). This suggests that AR proteolysis acts upstream of caspase activation, which leads to cell death. Although MG-132 alone slightly reduced AR protein levels in non-UV-irradiated cells through an unknown mechanism (Figure 1C), it did not affect cell viability (Figure 1D). Taken together, these results suggest that UV-induced proteasomal degradation of AR contributes to UV-induced cell death in prostate cancer $\mathrm{LNCaP} 104-$ $\mathrm{R} 1$ cells.

A

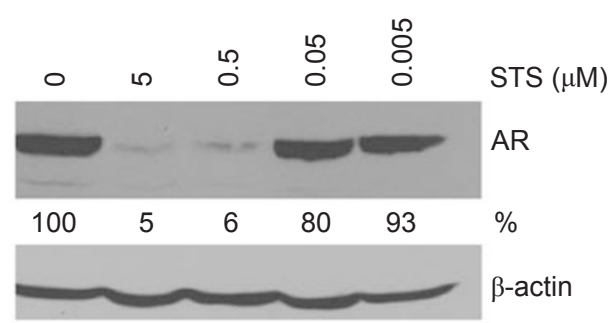

B

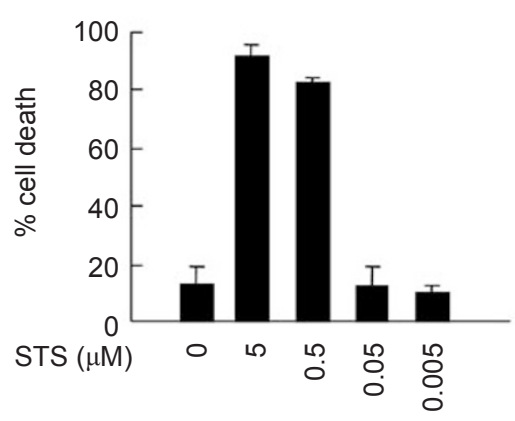

To determine whether AR degradation can be induced by other stresses, 104-R1 cells were treated with various doses of staurosporine (STS) for $8 \mathrm{~h}$, and degradation of AR was analyzed by immunoblotting using antiAR antibody (Figure 2A). We found that degradation of AR was induced by STS at a concentration as low as 0.5 $\mu \mathrm{M}$ (Figure 2A), corresponding to the dose of STS that induced cell death (Figure 2B). To determine whether AR degradation is involved in STS-induced cell death, 104$\mathrm{R} 1$ cells were pre-treated with MG-132 $(10 \mu \mathrm{M})$ for $2 \mathrm{~h}$, followed by treatment with STS $(0.5 \mu \mathrm{M})$ for various periods of time. MG-132 itself slightly reduced AR protein levels in non-treated cells, consistent with our previous finding (Figure 1C). However, STS-induced degradation of AR was significantly attenuated by MG-132 (Figure 2C). Under the same condition, STS-induced cell death was significantly inhibited by MG-132 (Figure 2D). Taken together, these data suggest that proteasomal degrada-

C
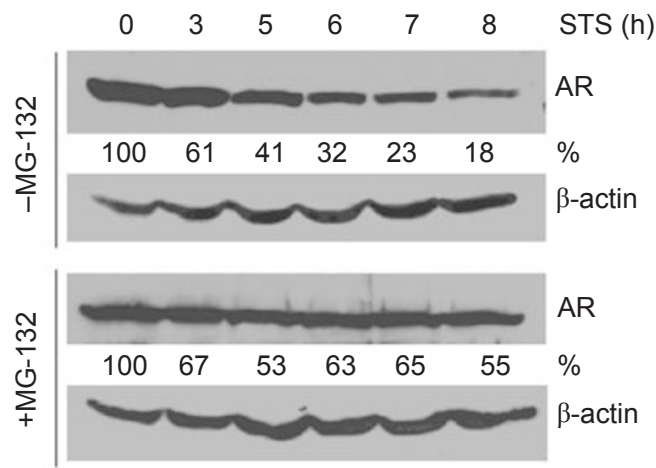

D

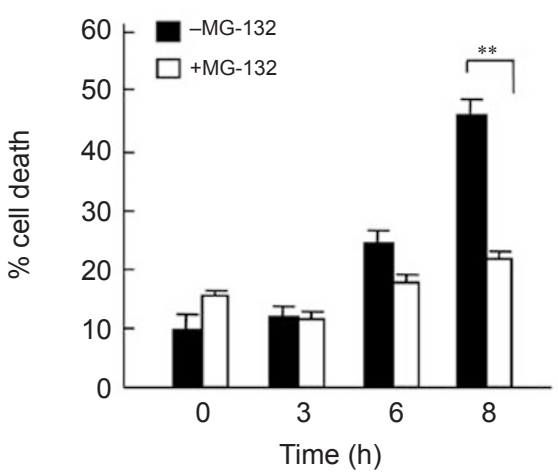

Figure 2 Proteasomal degradation of AR also promotes staurosporine-induced apoptosis. (A and B) LNCaP 104-R1 cells were treated with or without various doses of STS (staurosporine), as indicated for $8 \mathrm{~h}$. AR protein levels were analyzed by immunoblotting using anti-AR antibody. The percentage of AR reduction was normalized by $\beta$-actin (A) and cell death was monitored by trypan blue exclusion (B). (C and D) 104-R1 cells were pre-treated with MG-132 (10 $\mu$ M) for 2 h before STS (0.5 $\mu \mathrm{M})$ treatment, followed by incubation for various periods of time, as indicated. Cell death and AR expression were analyzed as in $\mathbf{A}$ and $\mathbf{B}$. Error bars indicate SD. ${ }^{*} P<0.01$ by $t$-test. Data shown are representative of two (A, C) or three (B, D) experiments. 
tion of AR also contributes to STS-induced cell death.

\section{The AR-N domain is sufficient to induce cell death}

The above observations prompted us to hypothesize that there may exist AR sub-domains with pro-death and transcription-independent activity, which are unleashed as a result of AR degradation. Since we were unable to detect the proteolytic fragments of AR (data not shown), we generated a series of truncated AR N-terminal mutants to test this hypothesis. To this end, androgenindependent, AR-negative prostate cancer PC-3 cells, which are resistant to UV-induced cell death [22], were transfected with expression vectors encoding full-length AR fused with green fluorescence protein (AR-GFP), AR-N-GFP, which lacks both DNA- and ligand- binding domains (Figure 3A), or with GFP alone, followed by UV irradiation or no treatment. Immunoblotting analysis of untreated cells revealed that expression levels of ARGFP and AR-N-GFP were comparable, as measured by anti-AR antibody (Figure 3B). Cell death assays showed that PC-3 cells transfected with GFP alone were resistant to UV-induced cell death, consistent with the previous report [22]. By contrast, PC-3 cells expressing AR-GFP had $15 \%$ cell death, which was further increased to $31 \%$ after UV irradiation (Figure 3C). Interestingly, ectopic expression of AR-N-GFP alone, without UV treatment, induced $30 \%$ cell death, similar to that induced by UV in the presence of AR-GFP (Figure 3C). More importantly, its pro-death activity was not augmented by UV irradiation (Figure 3C). Taken together, AR-N region appears to have constitutive pro-death activity that is sufficient to induce cell death in the absence of any additional death stimuli.

\section{Identification of pro- and anti-death domains in the AR-N region}

To identify the minimal pro-death domain(s) in AR-N region, AR-negative prostate cancer PC-3 cells were transfected with AR-N-GFP and further truncated mutants (Figure 4A, left panel). Cell death assays of GFPpositive cells revealed that the pro-death activity of the parent AR-N-GFP, AR(1-564), was further enhanced by the deletion of 129 amino acids at its $\mathrm{C}$-terminus (Figure 4A, right panel, compare AR(1-564) to AR(1435)). This suggests that an anti-death domain may be located between amino acids 436 and 564 . Interestingly, further deleted mutants, AR(1-307) and AR(1-202), almost completely lost their pro-death activities (Figure $4 \mathrm{~A}$, right panel). This indicates that a pro-death domain may be located between amino acids 307 and 435. However, the pro-death activity was restored by the AR(1105) mutant (Figure 4A, right panel), suggesting that amino acids 105-307 may also be an anti-death domain. Since amino acids 105-307 overlap the AF1 domain of AR $[9,10]$, AF1 may have a potential anti-death function. Conversely, deletion of amino acids 1-85 in AR-N region completely abolished its pro-death activity (compare $\mathrm{AR}(86-435)$ to $\mathrm{AR}(1-435))$, indicating that amino acids 1-85 may be necessary for the pro-death activity of AR(1-435). In support of this notion, we found that

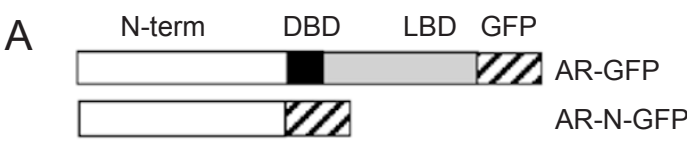

B

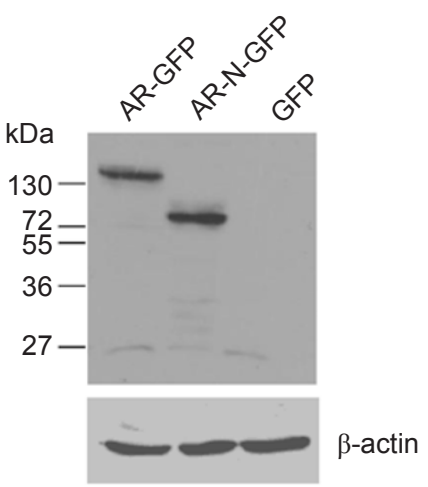

C

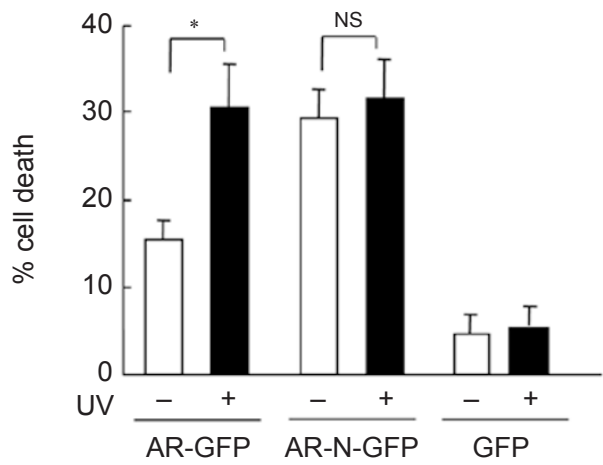

Figure 3 The AR N-terminal domain is sufficient to induce cell death. (A) cDNA containing full-length AR and the AR N-terminal region were tagged with GFP at their 3 ' ends. All constructs were sub-cloned into the expression vector pcDNA3.1 under the CMV promoter. (B) PC-3 cells were transfected with either GFP alone or GFP fusion constructs (AR-GFP or AR-N-GFP). At 48 $h$ after transfection, the expression of AR proteins was analyzed by immunoblotting using anti-AR antibody. (C) The death of transfected cells (GFP positive) was counted as described in Materials and Methods. Error bars indicate SD. ${ }^{*} P<0.05$; NS, no significant difference. Data are representative of three independent experiments. 
A

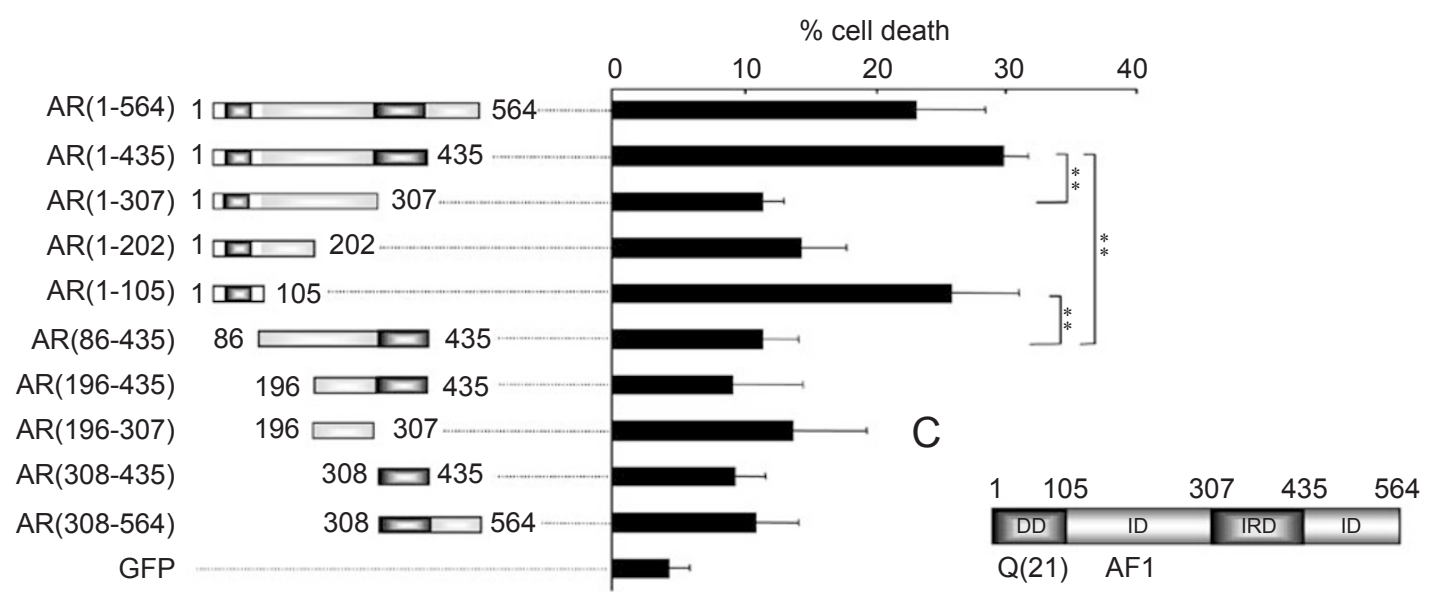

B

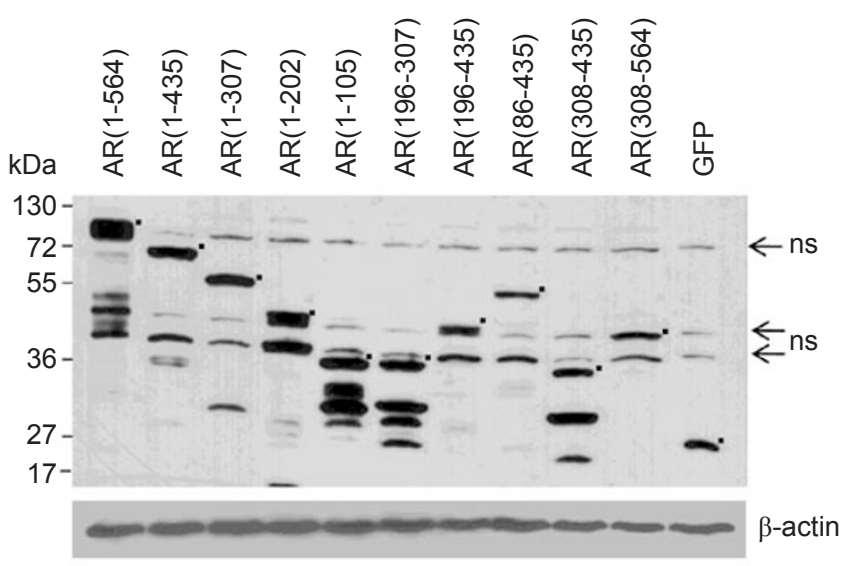

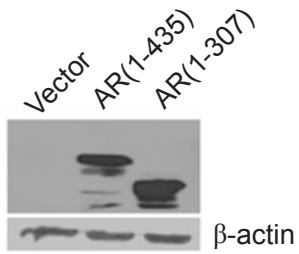

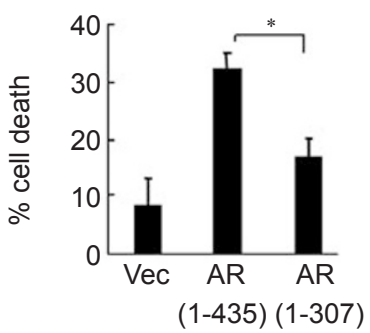

Figure 4 Identification of pro- and anti-death domains in the AR N-terminal region. (A) AR-negative PC-3 cells were transfected with expression vectors encoding various AR-GFP-truncated mutants or empty vector, as indicated (A, left panel). After $48 \mathrm{~h}$, cell death was measured for transfected GFP-positive cells (A, right panel). (B) The expression levels of AR proteins from A were analyzed by immunoblotting using anti-GFP monoclonal antibody. The positions of the AR proteins are indicated by dots. (C) A schematic illustration of AR functional domains. DD, death domain; ID, inhibitory domain; IRD, inhibitory release domain; $Q$, polyglutamine; and AF1, activation function-1 (transactivation) domain. (D) PC-3 cells were transfected with expression vectors encoding AR-truncated mutants, $A R(1-307)$ or $A R(1-435)$, or the empty vector. The expression levels of AR-truncated proteins were analyzed by immunoblotting with anti-AR antibody (upper panel). Cell death was measured 48 h post-transfection by trypan blue exclusion (lower panel). Transfection efficiency was generally more than $70 \%$. Error bars indicate SD. ${ }^{*} P<0.05$; ${ }^{* *} P<0.01$ by $t$-test. Data are representative of at least three independent experiments.

other AR truncation mutants, AR(86-435), AR(308-435), and AR(308-564), had no pro-death activity. Although AR(308-435) contains a pro-death domain, it was not sufficient to induce cell death (Figure 4A, right panel). It is likely that $\mathrm{AR}(308-435)$ serves as an inhibitory release domain (IRD), which releases the effect of the inhibitory domain (ID) located between amino acids 86 and 307 and thereby unleashes the pro-death activity of AR(1105). As illustrated in Figure 4C, the polyQ-rich AR(1105 ) functions as the minimal death domain (DD) and its pro-death activity was negatively or positively regulated by adjacent inhibitory domains (ID) and/or inhibitory release domains (IRD) within the AR-N region.

The pro-death activity of AR-truncated mutants was not due to their higher levels of expression, as measured by immunoblotting using anti-GFP antibody (Figure 4B). Similar results were obtained with AR-truncated mutants without the GFP tag. Like their GFP-tagged counterparts, AR(1-435) was pro-apoptotic, while AR(1-307) was not (Figure 4D). Thus, the intrinsic pro-apoptotic property of 
AR-N domain is unlikely due to its fusion to GFP.

To explore how the AR N-terminal fragments induce apoptosis, we examined the mode of apoptosis induced by the two most potent AR fragments, AR(1-435) and AR(1-105). We found that the pro-death activity of both AR fragments was blocked by ectopic expression of Bcl-2 (Figure 5A and 5B), suggesting that they may use the mitochondrial death pathway. Interestingly, the prodeath activity was also suppressed by the pan-caspase inhibitor z-VAD, as well as the caspase-8 inhibitor IETD, but not by the caspase-1 inhibitor YVAD (Figure 5C). This result indicates that these AR N-terminal fragments may act upstream of caspases, consistent with our finding of endogenous AR proteins in UV-treated LNCaP 104$\mathrm{R} 1$ cells (Figure 1C and 1D). Taken together, the AR-N region contains multiple pro- and anti-death domains (Figure 4C) that may determine the pro-death activity of AR through complex coordination in a caspase-dependent manner and subject to regulation by Bcl-2.

The pro-death activity of AR does not correlate with its aggregation ability

It has been reported that the polyQ repeats in AR proteins are responsible for forming aggregates and thereby
A

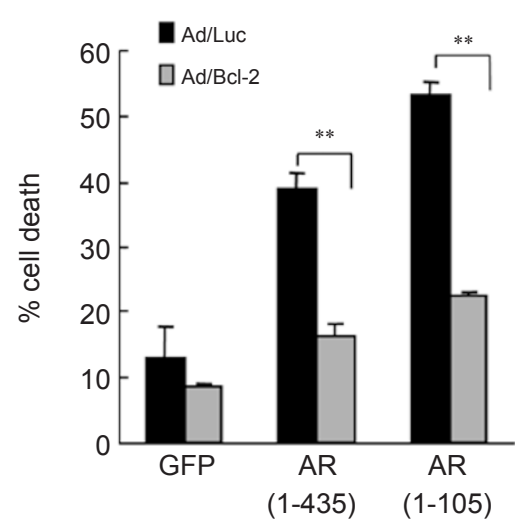

B

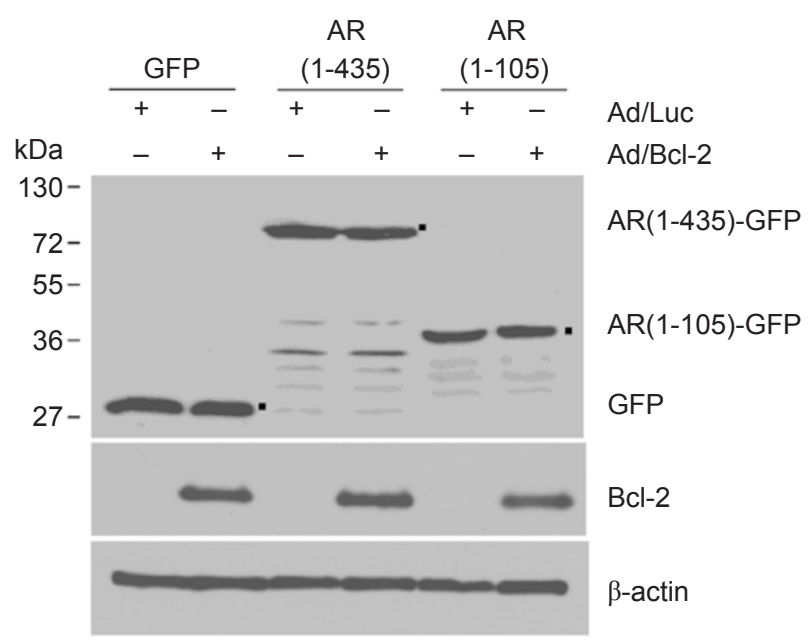

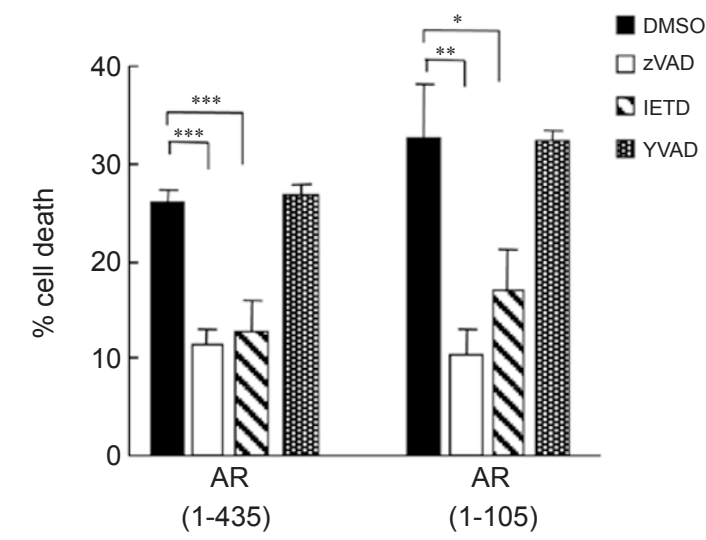

Figure 5 The pro-death activity of AR can be inhibited by Bcl-2 and selected caspase inhibitors. (A and B) AR-negative PC-3 cells were infected with adenovirus expressing Bcl-2 (Ad/Bcl-2) or luciferase (Ad/Luc). At $6 \mathrm{~h}$ after the infection, cells were transfected with GFP-AR pro-death mutants or the control GFP, as indicated. Cell death was measured for GFP-positive transfected cells $24 \mathrm{~h}$ post-transfection (A). The expression levels of GFP-AR or Bcl-2 proteins were measured by immunoblotting using anti-GFP and $\mathrm{Bcl}-2$ antibodies, respectively $(\mathrm{B})$. The positions of the expressed proteins are indicated by dots. (C) PC-3 cells were transfected with GFP-AR-truncated mutants or the control GFP in the absence or presence of different caspase inhibitors, as indicated. Cell death was monitored for green fluorescent cells. Error bars indicate SD. ${ }^{*} P<0.05 ; * * P$ $<0.01 ;{ }^{* *} P<0.001$ by $t$-test. Data are representative of two $(\mathbf{A}, \mathbf{B})$ and three $(\mathbf{C})$ independent experiments. 
producing cytotoxicity in cells $[24,28]$. Since the prodeath domain of AR(1-105) is rich in polyQ repeats (Figure 4C), we wondered whether the ability of AR-N-truncated mutants to form aggregates may correlate with the pro-death activity. To test this hypothesis, we examined the cellular localization of various AR-N-GFP-truncated mutants transfected in AR-negative PC-3 cells using confocal microscopy (Figure 6A). While expression of AR(1-435), AR(1-105), AR(196-435), and AR(1-307) resulted in similar degrees of aggregates (Figure 6A), only $\mathrm{AR}(1-435)$ and AR(1-105) were able to induce cell death (Figures 4A and 6B). Interestingly, AR(196-435) and AR(308-435), which lack polyQ repeats, were also able to form aggregates, but did not induce cell death (Figure $6 \mathrm{~A}$ and $6 \mathrm{~B}$ ). Thus, although cell death induced by some of the AR truncation mutants is accompanied by aggregation, the aggregation itself is not sufficient to induce cell death.

\section{Discussion}

Androgen receptor is known to have pro- or anti-death activity in prostate cancer cells, and its pro-death activity can be androgen-dependent or -independent [22, 29-31]. How AR promotes cell death independently of its transcription activity is incompletely understood. Here, we show that proteasomal degradation of AR is involved in stress-induced cell death in androgen-independent prostate cancer cells. Furthermore, ectopic expression of the AR-N region, which lacks both DNA- and ligand-binding domains, was able to constitutively induce cell death without any additional death stimuli. Thus, it is likely that stress-induced cell death in androgen-independent prostate cancer cells is mediated, at least in part, through proteasomal degradation of AR, and the proteolytic fragment(s) may in turn trigger cell death.

The finding that stress induces proteasomal degradation of AR and subsequent cell death uncovers a novel mechanism by which AR promotes stress-induced cell death independently of its transcriptional function [22]. However, how stress induces proteasomal degradation of AR remains to be determined. Since post-translational modifications such as phosphorylation and K48-linked polyubiquitination are often involved in degradation of
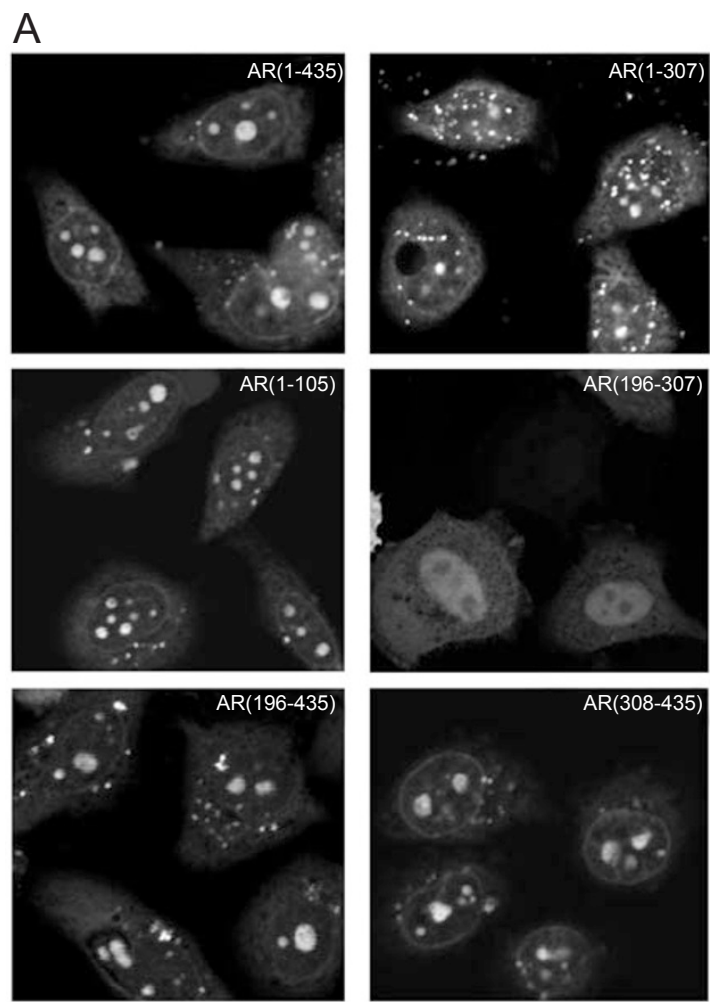

B

\begin{tabular}{lccc} 
Constructs & & Aggr. & Death \\
\cline { 1 - 1 } $\operatorname{AR}(1-564)$ & & + & + \\
$\operatorname{AR}(1-435)$ & & + & + \\
$\operatorname{AR}(1-307)$ & & + & - \\
$\operatorname{AR}(1-202)$ & & $\mathrm{ND}$ & - \\
$\operatorname{AR}(1-105)$ & & + & + \\
$\operatorname{AR}(196-307)$ & - & - \\
$\operatorname{AR}(196-435)$ & + & - \\
$\operatorname{AR}(86-435)$ & + & - \\
$\operatorname{AR}(308-435)$ & + & - \\
$\operatorname{AR}(308-593)$ & + & -
\end{tabular}

Figure 6 The pro-death activity of AR does not correlate with its aggregation. (A) AR-negative PC-3 cells were transfected with truncated GFP-tagged AR constructs for $16 \mathrm{~h}$. Cells were fixed, and the localization of GFP-tagged AR mutants was analyzed using a confocal microscope. (B) The degree of AR aggregation was scored by counting a minimum of 100 transfected cells. +, Aggregation positive or having significant cell death; -, aggregation negative or having no significant cell death; ND, not determined. 
target proteins by the proteasome, it is possible that UV or STS may induce post-translational modifications of AR to target it for proteasomal degradation. We are currently exploring these possibilities.

Our results that stress-induced cell death depends, at least in part, on proteasomal degradation of AR (Figures 1 and 2) is apparently contradictory to an early report that the proteasome inhibitor celastrol induces proteolysis of AR through activation of the protease, calpain, during apoptosis of LNCaP cells with no known mechanism [25]. Although calpain may play a role in AR proteolysis in celastrol-treated cells [25], this is unlikely the case for stress-induced AR degradation. We found that all three calpain inhibitors (calpain I, calpain II, and calpastatin) examined had no detectable effects on UV-induced AR degradation and cell death (data not shown). It is possible that different mechanisms are involved in UV- and calastrol-induced degradation of AR during cell death.

How AR proteolysis contributes to stress-induced cell death has yet to be determined. Although stress-induced proteasomal degradation of AR proteins was readily detectable (Figures 1B, 2A, and 2C) and was required for stress-induced cell death (Figures 1D and 2D), the proteolytic fragments of AR were undetectable for unknown reasons. Nevertheless, we found that AR-N domain-truncated mutants exhibited constitutive pro-death activities (Figures 3 and 4), consistent with a previous report that AR can be proteolyzed into several N-terminal domaincontaining fragments $(76 \mathrm{kDa}, 50 \mathrm{kDa}$, and $34 / 31 \mathrm{kDa}$ ) in a cell-free system [27]. Another possibility is that the full-length AR may have pro-survival function, which is eliminated by proteolysis. Future studies are needed to distinguish these possibilities.

The precise mechanism by which the AR N-terminal fragments induce cell death remains to be determined. Our results show that ectopic expression of Bcl-2 significantly suppressed the pro-death activity of AR Nterminal fragments (Figure 5A). This suggests that the pro-death activity of AR N-terminal fragments is, at least in part, mediated by the mitochondrial death pathway. This observation is consistent with our previous finding that full-length AR promotes UV-activated mitochondrial death pathway in prostate cancer cells [22]. Our results also show that pre-treatment with pan-caspase inhibitor z-VAD or caspase- 8 inhibitor, but not caspase-1 inhibitor, suppressed the pro-death activity of AR N-terminal fragments (Figure 5C). This suggests that the death receptor-dependent pathway is likely involved. However, it is unlikely that the AR fragments were able to activate the death receptors. It has been shown that the extended polyQ stretch is able to activate caspase-3, caspase-8, and caspase-9 through direct associations [32, 33]. Since deletion of the polyQ-rich domain in AR N-terminal fragments abrogated their abilities to induce cell death (Figure 4A), these fragments may use the polyQ-rich domain to target caspases downstream of the death receptor for cell death. Although it has been postulated that the polyQ-rich domain could induce aggregation of AR proteins, thereby triggering cell death $[24,28]$, our results show that the pro-death activity of AR did not correlate with its ability to aggregate (Figure 6).

The pro-death activity of AR is likely regulated by the death regulatory domains residing in its N-terminus, apart from its DNA- and ligand-binding domains. While AR(1-105) functions as the minimal death domain (DD), its pro-death activity was regulated by adjacent inhibitory domains (ID) and/or inhibitory release domains (IRD) within the AR-N region (Figure 4C). Future studies are needed to determine how the interplay between these regulatory domains determines the pro-death activity of $\mathrm{AR}$ in prostate cancer cells upon stress stimulation.

\section{Materials and Methods}

\section{Reagents and cell culture}

Anti-GFP antibody was obtained from Abcam (Cambridge, MA, USA). Anti-AR antibody (H280, which recognizes AR amino acids 91-370) was from Santa Cruz Biotechnology (Santa Cruz, CA, USA). Protease inhibitors leupeptin, aprotinin, benzamidine, and E64 were from Sigma. Calpain I and II, MG-132, and z-VADfmk were from Calbiochem (La Jolla, CA, USA). Human prostate cancer cells PC-3 were from ATCC, and LNCaP subline 104-R1 cells were generous gifts from Dr Shutsung Liao (The University of Chicago). Both cell lines were maintained in Dulbecco's modification of Eagle's medium supplemented with $10 \%$ dextrancoated, charcoal-stripped fetal bovine serum.

\section{Generation of AR-truncated mutants}

The full-length AR plasmid (pSG5-AR) was generously provided by Dr Shutsung Liao (The University of Chicago) and used as the template to generate all AR-truncated mutant constructs. All PCR-generated AR-truncated mutant constructs were sub-cloned into the expression vector pcDNA3.1. For generation of GFPtagged AR proteins, full-length GFP was in-frame engineered onto the C-terminus of each AR-truncated mutant construct and subcloned into pcDNA 3.1 vector. All constructs were verified by sequencing, and their expression was confirmed by immunoblotting with either anti-GFP antibody or anti-AR antibody.

\section{Transfection, viral infection, and cell death assays}

For transient transfection, cells were transfected using ExGen500 (Fermentas) according to the manufacturer's instructions, with typical transfection efficiency over $50-70 \%$. For cell death assays, cells were exposed to UV $\left(10 \mathrm{~mJ} / \mathrm{cm}^{2}\right)$ and then incubated at $37{ }^{\circ} \mathrm{C}$ for various periods of time, as indicated in the text. Cell death was detected by nuclear staining and trypan blue exclusion, or calculation of GFP-positive dead cells versus total GFPtransfected cells by directly counting or using a Flouroskan II fluo- 
rescent plate reader. For viral infection, cells were incubated with replication-deficient adenoviral vectors expressing either Bcl-2 (Ad/Bcl-2) or the control luciferase (Ad/Luc) at 100 MOI (multiplicity of infection) for $6 \mathrm{~h}$ before replacement of medium [34].

\section{Immunoblotting analysis and confocal microscopy}

Immunoblotting analysis was performed as described previously [22]. For detection of GFP-tagged AR-truncated proteins, PC-3 cells were grown on glass cover slides 1 day before transfection. Cells were fixed with pre-cold methanol for $7 \mathrm{~min}$ at room temperature. The slides were mounted and observed under a confocal microscope (Zeiss LSM5 Pascal).

\section{Acknowledgments}

We are grateful to Alex Bunce and Heather King for their work-study contributions as undergraduate students in the laboratory. This work is partially supported by the American Cancer Society (Illinois Division) and a grant from the National Institutes of Health (CA128114 to JX).

\section{References}

1 Kokontis JM, Hsu S, Chuu CP, et al. Role of androgen receptor in the progression of human prostate tumor cells to androgen independence and insensitivity. Prostate 2005; 65:287298.

2 Chang C, Kokontis J, Swift S, et al. Molecular cloning and structural analysis of complementary DNA of human and rat androgen receptors. Prog Clin Biol Res 1990; 322:53-63.

3 Chang CS, Kokontis J, Liao ST. Structural analysis of complementary DNA and amino acid sequences of human and rat androgen receptors. Proc Natl Acad Sci USA 1988; 85:72117215 .

4 Culig Z, Klocker H, Bartsch G, et al. Androgen receptors in prostate cancer. J Urol 2003; 170:1363-1369.

5 Chatterjee B. The role of the androgen receptor in the development of prostatic hyperplasia and prostate cancer. Mol Cell Biochem 2003; 253:89-101.

6 Georget V, Lobaccaro JM, Terouanne B, et al. Trafficking of the androgen receptor in living cells with fused green fluorescent protein-androgen receptor. Mol Cell Endocrinol 1997; 129:17-26.

7 Tomura A, Goto K, Morinaga H, et al. The subnuclear threedimensional image analysis of androgen receptor fused to green fluorescence protein. J Biol Chem 2001; 276:2839528401.

8 Liao SS, Kokontis J, Sai T, et al. Androgen receptors: structures, mutations, antibodies and cellular dynamics. J Steroid Biochem 1989; 34:41-51.

9 Chamberlain NL, Whitacre DC, Miesfeld RL. Delineation of two distinct type 1 activation functions in the androgen receptor amino-terminal domain. J Biol Chem 1996; 271:2677226778.

10 Lavery DN, McEwan IJ. Structure and function of steroid receptor AF1 transactivation domains: induction of active conformations. Biochem J 2005; 391:449-464.

11 Zhou ZX, Kemppainen JA, Wilson EM. Identification of three proline-directed phosphorylation sites in the human androgen receptor. Mol Endocrinol 1995; 9:605-615.

12 Nazareth LV, Weigel NL. Activation of the human androgen receptor through a protein kinase A signaling pathway. J Biol Chem 1996; 271:19900-19907.

13 Gioeli D, Ficarro SB, Kwiek JJ, et al. Androgen receptor phosphorylation. Regulation and identification of the phosphorylation sites. J Biol Chem 2002; 277:29304-29314.

14 Beitel LK, Elhaji YA, Lumbroso R, et al. Cloning and characterization of an androgen receptor N-terminal-interacting protein with ubiquitin-protein ligase activity. $\mathrm{J} \mathrm{Mol} \mathrm{Endocrinol}$ 2002; 29:41-60.

15 Poukka H, Karvonen U, Janne OA, et al. Covalent modification of the androgen receptor by small ubiquitin-like modifier 1 (SUMO-1). Proc Natl Acad Sci USA 2000; 97:14145-14150.

16 Claessens F, Denayer S, Van Tilborgh N, et al. Diverse roles of androgen receptor (AR) domains in AR-mediated signaling. Nucl Recept Signal 2008; 6:e008.

17 Risek B, Bilski P, Rice AB, et al. Androgen receptor-mediated apoptosis is regulated by photoactivatable androgen receptor ligands. Mol Endocrinol 2008; 22:2099-2115.

18 Isaacs JT. Apoptosis: translating theory to therapy for prostate cancer. J Natl Cancer Inst 2000; 92:1367-1369.

19 Heisler LE, Evangelou A, Lew AM, et al. Androgen-dependent cell cycle arrest and apoptotic death in PC-3 prostatic cell cultures expressing a full-length human androgen receptor. Mol Cell Endocrinol 1997; 126:59-73.

20 Young JE, Garden GA, Martinez RA, et al. Polyglutamineexpanded androgen receptor truncation fragments activate a Bax-dependent apoptotic cascade mediated by DP5/Hrk. $J$ Neurosci 2009; 29:1987-1997.

21 Yeh S, Hu YC, Rahman M, et al. Increase of androgeninduced cell death and androgen receptor transactivation by BRCA1 in prostate cancer cells. Proc Natl Acad Sci USA 2000; 97:11256-11261.

22 Lin Y, Kokontis J, Tang F, et al. Androgen and its receptor promote bax-mediated apoptosis. Mol Cell Biol 2006; 26:1908-1916.

23 Palazzolo I, Gliozzi A, Rusmini P, et al. The role of the polyglutamine tract in androgen receptor. J Steroid Biochem Mol Biol 2008; 108:245-253.

24 Ellerby LM, Hackam AS, Propp SS, et al. Kennedy's disease: caspase cleavage of the androgen receptor is a crucial event in cytotoxicity. J Neurochem 1999; 72:185-195.

25 Yang H, Murthy S, Sarkar FH, et al. Calpain-mediated androgen receptor breakdown in apoptotic prostate cancer cells. $J$ Cell Physiol 2008; 217:569-576.

26 Libertini SJ, Tepper CG, Rodriguez V, et al. Evidence for calpain-mediated androgen receptor cleavage as a mechanism for androgen independence. Cancer Res 2007; 67:9001-9005.

27 Sheflin L, Keegan B, Zhang W, et al. Inhibiting proteasomes in human HepG2 and LNCaP cells increases endogenous androgen receptor levels. Biochem Biophys Res Commun 2000; 276:144-150.

28 Kobayashi Y, Kume A, Li M, et al. Chaperones Hsp70 and Hsp40 suppress aggregate formation and apoptosis in cultured neuronal cells expressing truncated androgen receptor protein with expanded polyglutamine tract. J Biol Chem 2000; 275:8772-8778.

29 Niu Y, Altuwaijri S, Lai KP, et al. Androgen receptor is a tu- 
mor suppressor and proliferator in prostate cancer. Proc Natl Acad Sci USA 2008; 105:12182-12187.

30 Bhuiyan MM, Li Y, Banerjee S, et al. Down-regulation of androgen receptor by 3,3'-diindolylmethane contributes to inhibition of cell proliferation and induction of apoptosis in both hormone-sensitive $\mathrm{LNCaP}$ and insensitive $\mathrm{C} 4-2 \mathrm{~B}$ prostate cancer cells. Cancer Res 2006; 66:10064-10072.

31 Tepper CG, Vinall RL, Wee CB, et al. GCP-mediated growth inhibition and apoptosis of prostate cancer cells via androgen receptor-dependent and -independent mechanisms. Prostate 2007; 67:521-535.
32 U M, Miyashita T, Ohtsuka Y, et al. Extended polyglutamine selectively interacts with caspase- 8 and -10 in nuclear aggregates. Cell Death Differ 2001; 8:377-386.

33 Miyashita T, Matsui J, Ohtsuka Y, et al. Expression of extended polyglutamine sequentially activates initiator and effector caspases. Biochem Biophys Res Commun 1999; 257:724-730.

34 Xiang J, Gomez-Navarro J, Arafat W, et al. Pro-apoptotic treatment with an adenovirus encoding Bax enhances the effect of chemotherapy in ovarian cancer. J Gene Med 2000; 2:97-106. 